\title{
STUDY ON THE RESPONSE \\ CHARACTERISTICS OF AUTOMATIC WELDING FILTER
}

\author{
Huifang Liu ${ }^{1}$, Shiliang Jin ${ }^{1}$, Huiting Xiao ${ }^{1}$, Yue Hong ${ }^{1}$, Longii Shen ${ }^{2}$ \\ ${ }^{1}$ School of Mecanical Engineering, Shanghai University, China; Email:liudf-163@163.com \\ ${ }^{2}$ Department of Teaching, Shanghai University, China.
}

\begin{abstract}
To facilitate the application and development of the automatic welding filter, it is necessary to study its response characteristics. On the basis of the analysis and research about luminous transmittance and the scale number of the filter, this article proposes a solution to the specific definition of the response and holding time. By comparing different calculation methods in different standards, a felicitous way is chosen to describe the response characteristics accurately.
\end{abstract}

Key words: luminous transmittance; scale number; response time; holding time

\section{INTRODUCTION}

There are various hazards during the welding process, such as ultraviolet, infrared, organic gas, and so on. Until now, the welders usually use ordinary welding filter, it is harmful to their eyes. In China, lots of welders have ophthalmia every year. In this situation, the use of the automatic welding filter is extremely necessary.

The automatic welding filter has been used more and more extensively in modern society. It is a protective filter which automatically switches its scale number from a lower value (light state scale number) to a higher value (dark state scale number) when the welding arc is ignited, and protects the worker's eyes effectively so that the welding could be formed more efficiently and good quality is guaranteed ${ }^{[3]}$.

Please use the following format when citing this chapter:

Liu, Huifang, Jin, Shiliang, Xiao, Huiting, Hong, Yue, Shen, Longii, 2006, in International Federation for Information Processing (IFIP), Volume 207, Knowledge Enterprise: Intelligent Strategies In Product Design, Manufacturing, and Management, eds. K. Wang, Kovacs G., Wozny M., Fang M., (Boston: Springer), pp. 1016-1021. 
The structure of the automatic welding filter is as follows:

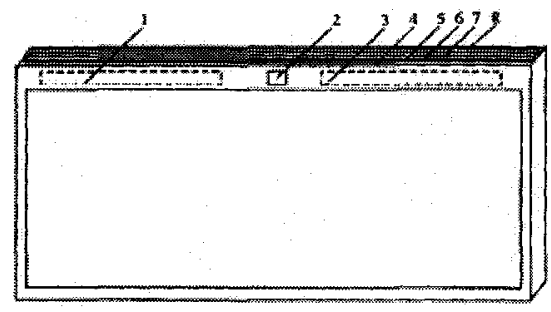

Figure 1. Structure of the automatic welding filter: 1 -control circuit, 2-light detector, 3electrical source, 4-frame, 5-outer protector, 6-ultraviolet and infrared filter, 7-light valve, 8 -inner protector.

\section{LUMINOUS TRANSMITTANCE AND THE SCALE NUMBER}

The luminous transmittance of a filter is the ratio of the transmitted spectral radiant flux $\phi_{\tau}$ to the incident spectral radiant flux $\phi_{i}$. In other words, $\tau=\frac{\phi_{\tau}}{\phi_{i}}$. To describe the response characteristics clearly, another parameter called scale number is introduced. The scale number consists of a coding number and a shade number: the coding number indicates the type of filter, with the exception of "welding filter without coding number"; the shade number is defined by the following expression:

$$
N=1+\frac{7}{3} \cdot \log _{10}\left(\frac{1}{\tau}\right)
$$

Here $\tau$ is the luminous transmittance. The shade number is the scale number for the filter that is studied in this article ${ }^{[5]}$.

To describe the state of the automatic welding filter in detail, it is better to divide the scale number into three parts, that is light state scale number, dark state scale number and darkest state scale number.

Light state scale number is the scale number corresponding to the maximum value of luminous transmittance $\tau_{1}$, dark state scale number is the scale number corresponding to the value of the luminous transmittance $\tau_{d}$ reached after the ignition of the welding arc by an automatic welding filter, and darkest state scale number is the highest scale number 
corresponding to the minimum value of luminous transmittance $\tau_{2}$ of an automatic welding filter claimed by the manufacture (see Figure 2).

$5 \quad 10 \quad 11 \quad 12 \quad 13 \quad 14$

Figure 2. Example showing the meaning of the terms in the case of an automatic welding filter with a light state scale number of 5 and dark state scale numbers between 10 and 14 .

The luminous transmittance requirements for filters used in welding and related techniques are given in Table 1.

Table I. Luminous transmittance requirements

\begin{tabular}{|c|c|c|c|c|c|}
\hline \multirow{2}{*}{$\begin{array}{l}\text { Scale } \\
\text { number }\end{array}$} & \multicolumn{2}{|c|}{$\begin{array}{l}\text { Luminous transmittance } \\
\tau\end{array}$} & \multirow{2}{*}{$\begin{array}{l}\text { Scale } \\
\text { number }\end{array}$} & \multicolumn{2}{|c|}{$\begin{array}{l}\text { Luminous transmittance } \\
\tau\end{array}$} \\
\hline & $\begin{array}{c}\text { Maximum } \\
\%\end{array}$ & $\begin{array}{c}\text { Minimum } \\
\%\end{array}$ & & $\begin{array}{c}\text { Maximum } \\
\%\end{array}$ & $\begin{array}{c}\text { Minimum } \\
\%\end{array}$ \\
\hline 1.2 & 100 & 74.4 & 8 & 0.16 & 0.061 \\
\hline 1.4 & 74.4 & 58.1 & 9 & 0.061 & 0.023 \\
\hline 1.7 & 58.1 & 43.2 & 10 & 0.023 & 0.0085 \\
\hline 2 & 43.2 & 29.1 & 11 & 0.0085 & 0.0032 \\
\hline 2.5 & 29.1 & 17.8 & 12 & 0.0032 & 0.0012 \\
\hline 3 & 17.8 & 8.5 & 13 & 0.0012 & 0.00044 \\
\hline 4 & 8.5 & 3.2 & 14 & 0.00044 & 0.00016 \\
\hline 5 & 3.2 & 1.2 & 15 & 0.00016 & 0.000061 \\
\hline 6 & 1.2 & 0.44 & 16 & 0.000061 & 0.000023 \\
\hline 7 & 0.44 & 0.16 & & & \\
\hline
\end{tabular}

\section{RESPONSE TIME AND HOLDING TIME}

There are two critical parameters for the automatic welding filter, one is the response time and the other is holding time. These two parameters determine whether the automatic welding filter being used is good or not.

Response time is the time from the light detector receiving the arc signal to the automatic welding filter turning to dark during the filter tested. It is not only related to the time in which the welders suffer from strong visible light, and it is also related to whether the welders can observe clearly during the welding process.

Holding time is the time from the light detector stopping the arc signal to the automatic welding filter turning back to its original state when the filter 
is in the dark state. During the welding process, it will happen 10 to 15 times instant short circuit per second, so the filter will change its state frequently if the holding time is too short. Then, the welders will be exposed to the strong visible light, this is bad to their health. Choosing a proper holding time is propitious to the continuous observation and the protection of the welder.

At present, based on the research provided above, different countries establish different standard definitions about the response characteristics of the automatic welding filter.

The European standard EN 379:2003 and the American standard ANSI Z87.1-2003 regulate that the response time of automatic welding filter is related to the filter's luminous transmittance and the scale number.

In these standards, the response time $t_{s}$ of an automatic welding filter is defined by the following integral:

$$
t_{s}=\frac{1}{\tau_{1}} \int_{t=0}^{t=t\left(\tau(t)=3 \tau_{2}\right)} \tau(t) d t
$$

Here: $\tau_{1}$ is the light state luminous transmittance; $\tau_{2}$ is the darkest state luminous transmittance; $t=0$ is the time at which the arc ignites; $\tau(t)$ is luminous transmittance at a time $t$ after the ignition of the welding $\operatorname{arc} ;=t\left(\tau(t)=3 \tau_{2}\right)$ is the time at which the luminous transmittance falls to 3 times the luminous transmittance in the darkest state.

But the Australian and New Zealand standard AS/NZS 1338. 1:1992 specifies the definition of response time and holding time in a different way. It records the response time as the time from the filter triggering as the shuttered light starts to rise in intensity, to the point where the filter reaches $90 \%$ of the dark state. And it records the holding time as the time from the filter triggering as the shuttered light starts to fall in intensity, to the point where the filter drops below $90 \%$ of the dark. In this standard, it stipulates that the minimum of the holding time should reach $20 \mathrm{~ms}^{[4]}$.

If we use luminous transmittance to define the holding time, the holding time will be the time from stopping the arc signal to the luminous transmittance reaches $90 \%$ of the light state.

From the above different definitions of the response characteristics we can get that the start point of the response time is uniform. Every standard begins the record with the light state (the luminous transmittance is maximum) when the filter receives the arc signal. And the start point of the holding time is the same too. They begin the record with the dark state (the luminous transmittance is minimum) when the filter eliminates the arc signal. However, there are differences among them, and they are the confirmation of the end point and the calculation method.

According to the analysis about response time and holding time above, we can easily get the conclusion: for the Australian and New Zealand 
standard, the definition of response and holding time is just related to the dark state intensity. They have nothing to do with the light state intensity. Furthermore, this definition just considers the beginning state and the ending state of the welding process, but doesn't consider the change during the test. It can't reflect the response characteristics timely, and the result won't be very precise. But for the European and American standards, the definition of response time not only has something to do with the light state luminous transmittance and dark state luminous transmittance of the automatic welding filter, but also reflects the change of the luminous transmittance during the welding. So, this definition can express the protection performance of the filter directly. Thus, the author suggests adopting the European and American definition to measure the response time. That is the formula (2).

As described above, it is better to adopt the time from stopping the arc signal to the luminous transmittance reaches $90 \%$ of the light state to measure the holding time. And also, for the health of the welder, the holding time should not be too short. It is recommended that the holding time should be more than $20 \mathrm{~ms}$, since the intensity of light is greatly reduced after the $20 \mathrm{~ms}$ of the extinguishments of the welding arc.

Different filters have different light state scale number and dark state scale number, so their response time is different. But for all of them, the response time given in Table 2 shall not be exceeded.

Table 2. Maximum response time

\begin{tabular}{|c|c|c|c|c|c|c|c|c|c|}
\hline \multirow{2}{*}{$\begin{array}{c}\text { Dark state scale } \\
\text { number }\end{array}$} & \multicolumn{7}{|c|}{ Light state scale number } \\
\cline { 2 - 8 } & 1.7 & 2 & 2.5 & 3 & 4 & 5 & 6 \\
\hline & \multicolumn{7}{|c|}{ Response time (ms) } \\
\hline 7 & 300 & 400 & 500 & 700 & 1000 & - & - \\
\hline 8 & 100 & 150 & 200 & 300 & 500 & 1000 & - \\
\hline 9 & 40 & 50 & 70 & 100 & 200 & 400 & 700 \\
\hline 10 & 20 & 20 & 30 & 40 & 70 & 100 & 300 \\
\hline 11 & 6 & 7 & 10 & 15 & 30 & 50 & 100 \\
\hline 12 & 2 & 3 & 4 & 5 & 10 & 20 & 40 \\
\hline 13 & 0.8 & 1 & 1.5 & 2 & 4 & 7 & 10 \\
\hline 14 & 0.3 & 0.4 & 0.5 & 0.7 & 1 & 3 & 5 \\
\hline 15 & 0.10 & 0.15 & 0.2 & 0.3 & 0.5 & 1 & 2 \\
\hline 16 & 0.04 & 0.05 & 0.07 & 0.1 & 0.2 & 0.4 & 0.7 \\
\hline
\end{tabular}

This is an example of the measurement. The range of the filter's scale number is 5 to 11 . It's response characteristics are described by the curve given in Figure 3. 


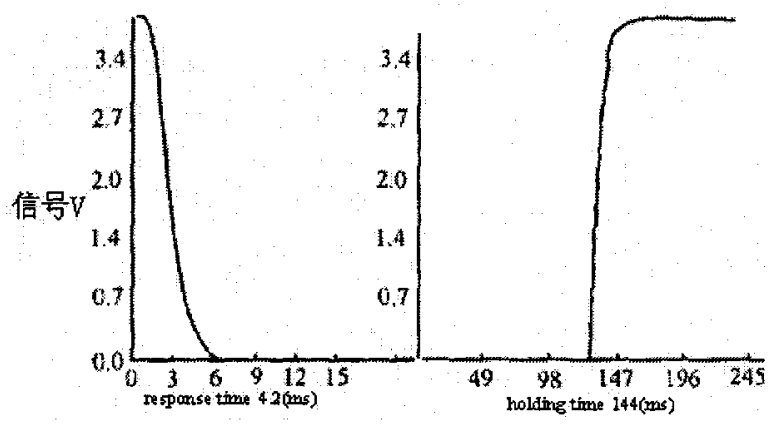

Figure 3. The curve of response characteristics.

\section{CONCLUSIONS}

According to the compare and research on the response characteristics of automatic welding filters in different countries, this article provides some new requirements and thoughts: the response characteristics have something to do with the luminous transmittance of the filter itself; the response characteristics should reflect the change of the state during the welding process, not just the start point and the end point; the response time is defined as formula (2), and the holding time should be more than $20 \mathrm{~ms}$. This research has great significance to the development and enlargement of new products, and it is very useful to evaluate the quality of new products too.

\section{REFERENCES}

1. Jeng Jeng-Ywan, Mau Tzuoh-Fei, Leu Shyeu-Ming. Prediction of laser butt joint welding parameters using back propagation and learning vector quantization networks. Journal of Materials Processing Technology Volume: 99, Issue: 1-3, March 1, 2000, pp. 207-218

2. Hsieh J.C., Liu C.J., Ju Y.H. Response characteristics of lead phthalocyanine gas sensor: effects of film thickness and crystal morphology. Thin Solid Films Volume: 322, Issue: 1-2, June 8, 1998, pp. 98-103

3. EN379: 2003, Personal Eye-protection-Automatic welding filters [S], 2003

4. AS/NZS 1338.1: 1992, Filters for eye protectors [S], 1992

5. ANSI Z87.1-2003 [S], 2003

6. EN 165:1995, Personal eye-protection-Vocabulary [S], 1995

7. ISO 4850-1979, Personal eye-protectors for welding and related techniques- FiltersUtilisation and transmittance requirements [S], 1979 